\title{
Studying Cell Physiology and Motility under Microgravitational Influence - Results of the FORAREX Mission on REXUS 25
}

\author{
Greta Sondej ${ }^{1}$, Nils Kunst ${ }^{1}$, Christoph Kulmann ${ }^{1}$, \\ Scarlett Gac Cáceres ${ }^{1}$, Diren Senger ${ }^{1}$, \\ Claudia Bruhn ${ }^{1,2}$, Katrin Hättig ${ }^{1}$, Sebastian Gfellner ${ }^{1}$, \\ Kay Menkens-Siemers ${ }^{1}$, Niklas Kipry ${ }^{1}$, \\ Daniel Ripberger ${ }^{1}$, Jan Blumenkamp ${ }^{1}$, \\ Steffen Lohmann ${ }^{1}$ and Johanna Hartmann ${ }^{1,3}$
}

\begin{abstract}
The student project "FORAminifera Rocket EXperiment" (FORAREX) was developed and conducted within the student educational programme REXUS/BEXUS. Focus of this investigation was the cellular response of foraminifera to microgravity and the exceptional physical stress during rocket launch, e.g., vibration and acceleration. Further the launch impact on the shell building capacity of foraminifera was examined. For cultivating purposes, a life-support-system with a flow cell as observation chamber was built. Monitoring vitality and calcification was conducted with sensors measuring $\mathrm{pH}$, oxygen and temperature of the sea water. FORAREX launched in March 2019 on the sounding rocket REXUS 25 on a nominal flight.
\end{abstract}

This paper has been submitted in a similar version elsewhere.

Keywords- Foraminifera, microgravity, cell physiology and motility, Gravitational Biology, life-support system, sounding rocket, REXUS/ BEXUS programme, International Space Station

\section{INTRODUCTION}

Foraminifera are mainly marine unicellular organisms which typically produce an external shell, which can have either one or multiple chambers. These shells are most commonly made of calcium carbonate $\left(\mathrm{CaCO}_{3}\right)$ or agglutinated sediment particles. Foraminifera are characterised by streaming granular ectoplasm through their aperture called granuloreticulopodia, hereinafter referred as pseudopodia. The latter consists of cytoskeleton structures representing a multifunctional cellular network serving various purposes such as locomotion, feeding, digestion, and chamber formation [1], [2], [3].

First studies on mice show that foraminiferal shells can be employed as material for drug delivery systems, which allows for a better control of drug release than conventional applications [4]. Another important application is the use of the shells in bone revitalisation already tested in mice [4], [5]. Biominerals derived from marine organisms are an interesting field of research for nano-scale chemical reactions. The calcium carbonate can be coupled with different substrates,

\author{
${ }^{1}$ University of Bremen, 28359 Bremen, Germany \\ ${ }^{2}$ Alfred Wegener Institute for Polar and Marine Research, \\ 27570 Bremerhaven, Germany \\ ${ }^{3}$ University of the Arts Bremen, 28217 Bremen, Germany \\ Corresponding authors' email addresses: \\ greta.sondej@uni-bremen.de,nkunst@uni-bremen.de
}

possibly enabling improved or even new manufacturing and processing methods [6], [7], [8], [9], [10], [11].

Crystallisation of biomolecules is already known to be different under microgravity than on Earth. For example, albumin crystals formed on board of the International Space Station (ISS) were significantly larger and more regularly organised compared to the same crystals formed under Earth gravity conditions [12]. As calcareous foraminiferal shells are compounds of proteins and calcite, we expect the crystallisation in their shell biomineralisation process to be affected in a similar way under microgravity. The complete process is not yet fully understood and thus an interesting field of research. This opens up the creation of new biomaterials with possible applications in drug development, formulation, manufacturing and medicine.

As preceding investigation for a long-term experiment on board of the ISS, where we would like to investigate foraminiferal shell formation and cell physiology under extended microgravity influence, the FORAREX experiment was developed and conducted in the course of the REXUS/BEXUS programme - an opportunity for university students to carry out scientific and technological experiments on board of sounding rockets or stratosphere balloons. The programme is realised under a bilateral Agency Agreement between the German Aerospace Center (DLR) and the Swedish National Space Agency (SNSA). The Swedish share of the payload has been made available to students from other European countries through the collaboration with the European Space Agency (ESA). Experts from DLR, SSC, ZARM and ESA provide technical support to the student teams throughout the project. EuroLaunch, the cooperation between the Esrange Space Center of SSC and the Mobile Rocket Base (MORABA) of DLR, is responsible for the campaign management and operations of the launch vehicles [13], [14].

Under the limited microgravity duration provided by the REXUS sounding rockets, we focused on testing the equipment and the motility of the foraminifera Amphistegina lobifera Larsen 1976 [15] during launch and flight in comparison to a control group. 
Since microgravity induces changes in cytoskeletal structures [16], it is interesting to investigate if the pseudopodia of the living cell would change under microgravity, as the shell of foraminifera is synthesised using the pseudopodia as a template. Additionally, the shell building capability and motility was investigated by optical means, scanning electron microscopy (SEM) and nanoscale secondary ion mass spectrometry (nanoSIMS) after 4 weeks post-flight cultivation. For improved shell analysis we labelled the shells with strontium isotopes. For this experiment, we designed and constructed a Late Access Module (LA Module) accommodating the closed-circuit life-support system with integrated LED-based illumination for real-time observations of foraminifera and tested it successfully under reduced gravity and sounding rocket mission conditions. Low-drift optical sensors continuously measured oxygen $\left(\mathrm{O}_{2}\right)$ and $\mathrm{pH}$ level. Water temperature and ambient pressure were measured with electronic sensors. Foraminifera were recorded with integrated and automated Raspberry Pi cameras.

\section{MATERIAL AND METHODS}

\section{A. Life-support system}

The designed life-support system consisted of two independent water circulation cycles. Each cycle system contained one flow cell to hold the organism, UV-lights for sterilisation, a water tank, a peristaltic pump, LEDs for photosynthetic active radiation (PAR), a camera and sensors for measuring oxygen concentration, temperature and $\mathrm{pH}$ level (Fig. 1). The system was controlled by a Raspberry Pi Compute Module 3.

A minicomputer read out the cameras (one for each chamber) and the sensor values $\mathrm{O}_{2}$ and $\mathrm{pH}$ level for water cycle 0 (Exp0) and $\mathrm{O}_{2}$ for water cycle 1 (Exp1). Further it read out temperature, air pressure and values of an inertial measurement unit (IMU). Moreover it controlled the actuators (pumps, heater, fan and LEDs (PAR and UVC)). Furthermore a real time clock for better time references and temperature sensors for active temperature regulation was integrated.

Each flow cell functioned as an observation chamber for one single foraminifer. Injection of the organism was done via a cannula through layers of septa at one side of the flow cell. This was necessary for injection of the foraminifera into the fully assembled LA Module shortly prior to lift-off (LO).

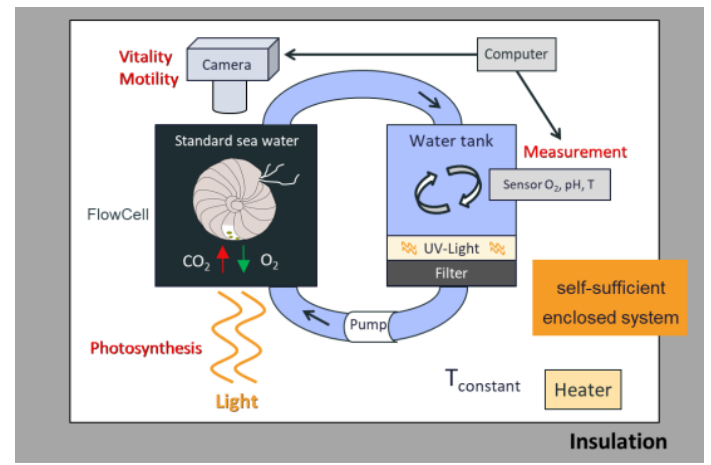

Fig. 1. Schematic diagram of one water cycle. Each water cycle is a selfsufficient enclosed system.

\section{B. Experimental set-up}

Foraminifera $1.3 \mathrm{~mm}$ in diameter were used. This ensured visibility of the organisms and their pseudopodia. The foraminifera were kept in artificial seawater (Tropic Marin ${ }^{\circledR}$ CLASSIC SEA SALT, according to Standard ASTM D114198) at $20^{\circ} \mathrm{C}$ under a day-night cycle of 12 hours light and 12 hours darkness. The simulation of the diurnal rhythm was conducted with light according to the required photosynthetic light spectrum of the endosymbiotic algae (diatoms Fragilaria shiloi J. J. Lee, Reimer \& McEnery 1980 [17] and Nitzschia panduriformis Gregory 1857 [18]). Food for the foraminifera was omitted in order to stimulate foraging movements of the cells.

Supplementation of the culture medium with strontium was necessary in order to distinguish the shell sections that have been grown before, during and after the rocket flight phase. A first chemical labeling with strontium chloride-hexahydrate $\left(\mathrm{SrCl}_{2} * 6 \mathrm{H}_{2} \mathrm{O}\right)$ was added to the sea water 6 days before launch. Strontium-86 carbonate isotope $\left({ }^{86} \mathrm{SrCO}_{3}\right)$ was added as a second label the day after flight.

The cells were inserted into the flow cell 2 days before rocket launch. The LEDs placed directly above the foraminifera supplied them with light for photosynthesis and illuminated them when a picture was taken by the cameras. Pictures were recorded every 2 seconds and sensor data were recorded every 5 seconds before, during and after flight.

After recovery, the experiment was run for additional 31 days at $20^{\circ} \mathrm{C}$ in an incubator and then disassembled. Cells were evaluated for evidence of life in petri dishes with freshly added food. Shell integrity of the foraminifera was evaluated by means of light microscopy.

A ground control group was conducted in the same experiment procedure apart from the rocket launch and flight.

\section{Sounding rocket and flight}

The REXUS vehicle is a one-stage rocket, consisting of an improved Orion motor and the payload. This rocket gives approximately two minutes of microgravity conditions with a payload mass of up to $\sim 95 \mathrm{~kg}$, including the service and recovery systems [14]. REXUS 25 was launched on $11^{\text {th }}$ of March, 2019 at 10:20:00 local time (LO UTC+1:00) with five student experiments (FLOMESS, HEDGEHOG, $\mathrm{PR}^{3}$, FORAREX, GAME) on board from the European Space and Sounding Rocket Range (Esrange) near Kiruna, Sweden. The total weight of the sounding rocket setup was $541 \mathrm{~kg}$ at a size of $5.927 \mathrm{~m}$.

The LA Module was prepared shortly before launch and was inserted into the rocket 2 hours 50 minutes before LO. The successful connection was checked via the uplink/downlink communication.

Rocket motor burnout was after $\mathrm{T}+26 \mathrm{~s}$. At $\mathrm{T}+67 \mathrm{~s}(67$ seconds after LO) yo-yo de-spin was set in shortly followed by the rocket motor ejection at $\mathrm{T}+70 \mathrm{~s}$. Apogee was reached after $\mathrm{T}+124 \mathrm{~s}$ at a minimum altitude of $82.184 \mathrm{~km}$. Parachutes were deployed at $\mathrm{T}+5 \mathrm{~min} 55 \mathrm{~s}$ and $\mathrm{T}+6 \mathrm{~min} 20 \mathrm{~s}$ and the rocket landed at $\mathrm{T}+13 \mathrm{~min} 41 \mathrm{~s}$. 


\section{RESULTS}

As the launch was scheduled for early morning, the LA Module was prepared a day ahead and was completed in time. All the systems of the experiment operated as planned during the nominal flight.

Water temperature increased up to $31^{\circ} \mathrm{C}-34^{\circ} \mathrm{C}$ during flight (Fig. 2, 3). Oxygen concentration in both water cycles dropped sharply a few minutes after LO (Fig. 4, 5). Pressure within the flight module dropped from 1,000 to under $600 \mathrm{hPa}$ at LO (Fig. 7). Alkalinity spiked from $\mathrm{pH} 7.09$ to $\mathrm{pH} 7.13$ at LO, then stabilised at $\mathrm{pH} 7.11$ during parachute breaking and landing (Fig. 6).

After a successful recovery, the LA Module was brought back to the laboratory to supply it with electricity for the postflight data recording. Both water cycles showed an initiate temperature of $14^{\circ} \mathrm{C}-15^{\circ} \mathrm{C}$.

In-depth analysis of the post-flight data as well as the launch and microgravity impact on the shell biomineralisation will be published in separate papers.

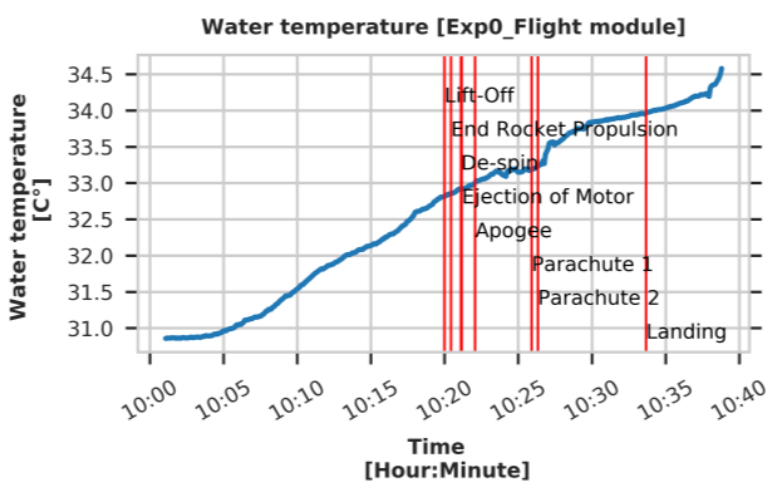

Fig. 2. Water temperature of Exp0. LO at 10:20:00 local time (UTC+1:00).

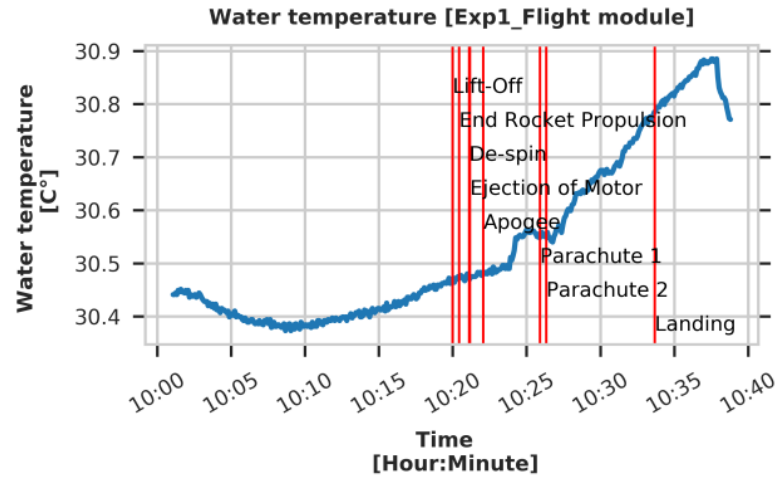

Fig. 3. Water temperature of Exp1. LO at 10:20:00 local time (UTC+1:00).

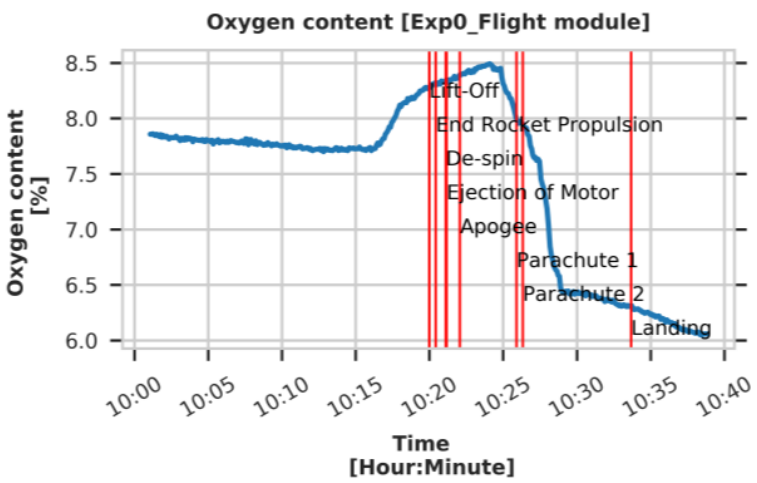

Fig. 4. Oxygen content of Exp0. LO at 10:20:00 local time (UTC+1:00).

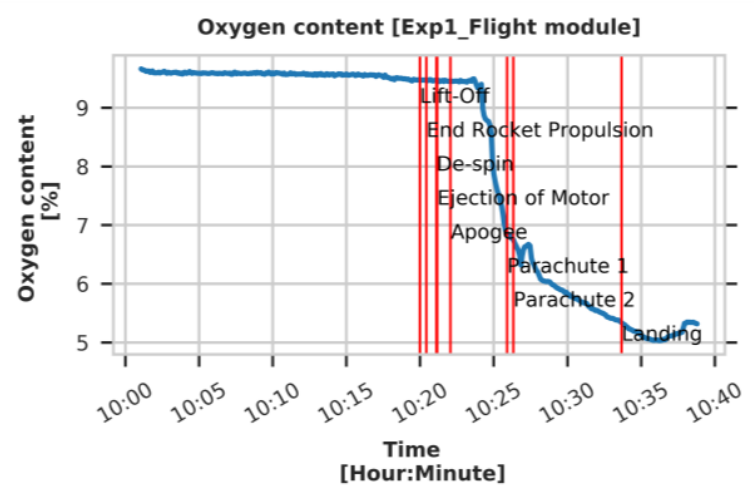

Fig. 5. Oxygen content of Exp1. LO at 10:20:00 local time (UTC+1:00).

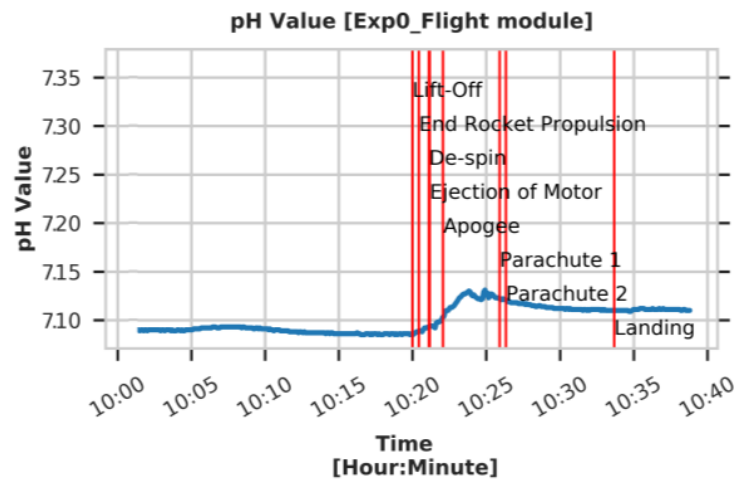

Fig. 6. pH Value of Exp1. LO at 10:20:00 local time (UTC+1:00). 


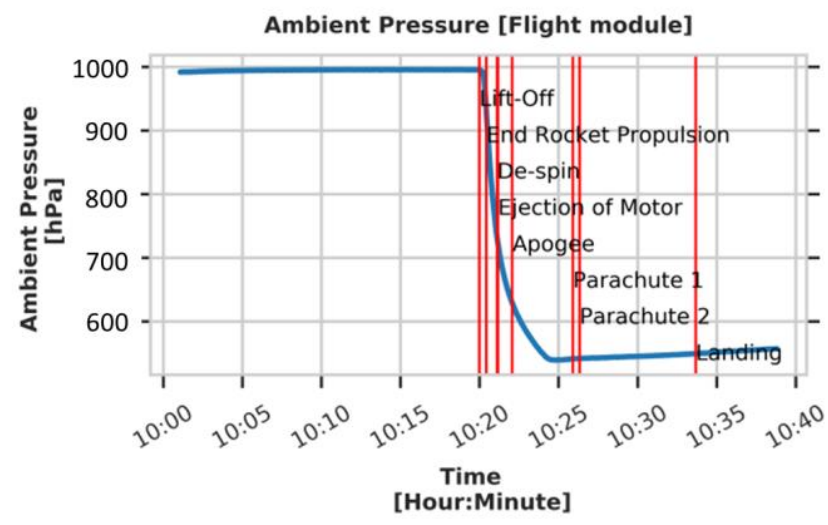

Fig. 7. Ambient pressure inside the LA module. LO at 10:20:00 local time (UTC+1:00).

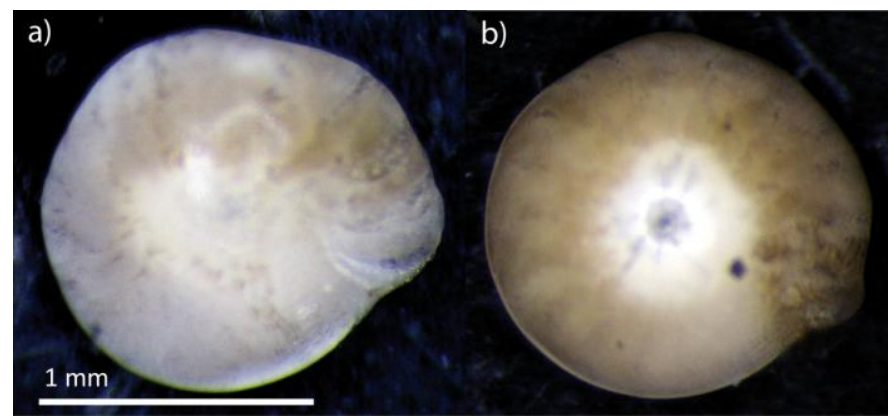

Fig. 8. Specimens of Amphistegina lobifera used in this experiment. Scale bar is $1 \mathrm{~mm}$. a) Foraminifer in water cycle $0(\operatorname{Exp} 0)$, b) foraminifer in water cycle 1 (Exp1).

\section{DISCUSSION AND CONCLUSIONS}

Cameras and monitoring sensors worked within the limits of their specifications, allowing the observation of living foraminifera during rocket flight. The increased water temperature before flight derived from the heated environment $\left(30^{\circ} \mathrm{C}-31^{\circ} \mathrm{C}\right)$, especially applied by the ground operation crew for this mission to prevent freezing of the foraminifera, before the rocket was brought into launch position. The insulated LA Module kept the water temperature within range until landing.

The major drop in oxygen concentration in both water cycles is presumably due to the drop of the ambient pressure within the LA Module. This may have caused some outgassing of dissolved atmospheric gases (nitrogen, oxygen and carbon dioxide), as the solution of gases in water is pressuredependent. Outgassing of carbon dioxide may be indicated by a slight shift in alkalinity shortly after lift-off. During parachute breaking and landing, the carbon dioxide redissolved in the water due to increasing ambient pressure and restored the original alkalinity by forming carbonic acid.

In summary, our life-support system and LA Module were suitable to observe living foraminifera during rocket flight.

\section{ACKNOWLEDGMENT}

We thank our supervisor Professor Dr. Michal Kucera and his working group from the Center for Marine Environmental Sciences (MARUM) of the University of Bremen for providing us with Amphistegina lobifera and helping us with their expertise. We also would like to thank the Electronics Workshop of the working group on Microsensors of the MaxPlanck-Institute of Marine Microbiology and Professor Dr. Jelle Bijma and his working group on of Marine BioGeoScience of the Alfred Wegener Institute, Helmholtz Centre for Polar and Marine Research for counselling interviews. To our consulting and material supporters from the aerospace sector Airbus, the German Research Center for Artificial Intelligence - Robotics Innovation Center (DFKI GmbH RIC Bremen) and the OHB Systems AG we would like to express our sincere thanks.

Further we would like to express the special gratitude of our industrial partners BuyIsotope Neonest AB, OMNILABLABORZENTRUM GmbH \& Co.KG, PreSens Precision Sensing $\mathrm{GmbH}$, PyroScience $\mathrm{GmbH}$, Watson-Marlow $\mathrm{GmbH}$ Fluid Technology Group and ZF Friedrichshafen AG (training centre Lemförde).

We would like to offer our best thanks to Dr. Hans-Konrad Nettmann and Professor Dr. Karl-Heinz Blotevogel for scientific advice and logistic services. A big thank you goes to Timothy Sondej for helping with Python scripting and consulting in data analysis.

Finally, we seize this opportunity to extend our deepest thanks and appreciation for the REXUS/BEXUS programme and its supporting agencies, namely the German Aerospace Center (DLR), the Swedish National Space Agency (SNSA), Swedish Space Corporation (SSC), European Space Agency (ESA) and the ZARM Drop Tower Operations and Service Company for their outstanding support to make this project possible.

\section{REFERENCES}

[1] Erez, J. (2003). The source of ions for biomineralization in foraminifera and their implications for paleoceanographic proxies. Reviews in mineralogy and geochemistry, 54(1), 115-149.

[2] Nagai, Y., Uematsu, K., Chen, C., Wani, R., Tyszka, J., \& Toyofuku, T. (2018). Weaving of biomineralization framework in rotaliid foraminifera: implications for paleoceanographic proxies. Biogeosciences, 15(22), 6773.

[3] Murray, J. W. (2006). Ecology and applications of benthic foraminifera Cambridge University Press.

[4] Chou, J., Ito, T., Otsuka, M., Ben-Nissan, B., \& Milthorpe, B. (2013). Simvastatin-Loaded $\beta$-TCP drug delivery system induces bone formation and prevents rhabdomyolysis in OVX mice. Advanced Healthcare Materials, 2(5), 678-681.

[5] Chou, J., Hao, J., Kuroda, S., Bishop, D., Ben-Nissan, B., Milthorpe, B., \& Otsuka, M. (2013). Bone regeneration of rat tibial defect by zinctricalcium phosphate (zn-TCP) synthesized from porous foraminifera carbonate macrospheres. Marine Drugs, 11(12), 5148-5158.

[6] Green, D. W., Ben-Nissan, B., Yoon, K. S., Milthorpe, B., \& Jung, H. (2017). Natural and synthetic coral biomineralization for human bone revitalization. Trends in Biotechnology, 35(1), 43-54. 
[7] Clarke, S. A., Walsh, P., Maggs, C. A., \& Buchanan, F. (2011). Designs from the deep: Marine organisms for bone tissue engineering. Biotechnology Advances, 29(6), 610-617.

[8] Shi, Jiafu, et al. "Design and synthesis of organic-inorganic hybrid capsules for biotechnological applications." Chemical Society Reviews 43.15 (2014): 5192-5210.

[9] Elhadj, S., Salter, E. A., Wierzbicki, A., De Yoreo, J. J., Han, N., \& Dove, P. M. (2006). Peptide controls on calcite mineralization: Polyaspartate chain length affects growth kinetics and acts as a stereochemical switch on morphology. Crystal Growth \& Design, 6(1), 197-201.

[10] Davis, S. A., Breulmann, M., Rhodes, K. H., Zhang, B., \& Mann, S. (2001). Template-directed assembly using nanoparticle building blocks: A nanotectonic approach to organized materials. Chemistry of Materials, 13(10), 3218-3226.

[11] Sandhage, Kenneth Henry, and Zhihao Bao. "Methods of fabricating nanoscale-to-microscale structures." U.S. Patent No. 7,615,206. 10 Nov. 2009.

[12] DeLucas, Lawrence J., et al. "Protein crystal growth in microgravity." Science 246.4930 (1989): 651-654.
[13] Callens, N., et al. "REXUS/BEXUS-Rocket and balloon experiments for university students." 21st ESA Symposium on European Rocket \& Balloon Programmes and Related Research. 2013.

[14] Schüttauf, K., et al. (2018) EuroLaunch REXUS User Manual, Document ID: RX_UserManual_v7-16_24Oct18

[15] Hayward, B.W.; Le Coze, F.; Vachard, D.; Gross, O. (2019). World Foraminifera Database. Amphistegina lobifera Larsen, 1976.

[16] Corydon, Thomas J., et al. "Alterations of the cytoskeleton in human cells in space proved by life-cell imaging." Scientific reports 6 (2016): 20043.

[17] Lee, J. J., Reimer, C. W., \& McEnery, M. E. (1980). The Identification of Diatoms Isolated as Endosymbionts from Larger Foraminifera from the Gulf of Eilat (Red Sea) and the Description of 2 New Species, Fragilaria shiloi sp. nov. and Navicula reissii sp. nov. Botanica marina, 23(1), 41-48.

[18] Gregory, W. (1857). On new forms of marine Diatomaceae found in the Firth of Clyde and in Loch Fyne, illustrated by numerous figures drawn by K.K. Greville, LL. D., F. R. S. E. Transactions of the Royal Society of Edinburgh, 21: 473-542, pls. 9-14. 